\title{
Upper airway obstruction in the cat
}

\section{Lynelle Johnson}

Cats can develop clinical signs associated with obstruction of the nasal cavity, nasopharynx, larynx and trachea. The most common nasal causes of obstruction include nasal neoplasia, fungal infection, nasopharyngeal polyp and nasopharyngeal stenosis. Nasal discharge is not always present but stertorous respiration is common. Affected cats generally have decreased nasal airflow unilaterally or bilaterally on physical examination. Cats with bilateral nasal or nasopharyngeal disease can breathe well through the mouth, but exhibit distress when the mouth is held closed. Whenever possible, the caudal aspect of the soft palate should be palpated for abnormalities. Generally, the soft palate is easily depressed into the roof of the nasopharynx with digital palpation. A nasopharyngeal mass (tumour, granuloma or polyp) can be felt as a space-occupying lesion dorsal to the soft palate. A lateral cervical radiograph that includes the caudal aspect of the mandible can be helpful in defining a mass lesion. Fungal infection can be documented with cryptococcal antigen testing (CALAS or LCAT). Nasal neoplasia and nasopharyngeal stenosis generally require advanced imaging (computed tomography (CT)) and rhinoscopy (caudal and rostral) with biopsy for diagnosis.

Cats with laryngeal disease can present for inspiratory difficulty, abnormal purr or loss of meow. Gagging and decreased appetite can occur. Causes include a laryngeal mass (lymphoma or squamous cell carcinoma), inflammatory laryngitis or laryngeal paralysis. A lateral cervical radiograph or laryngeal ultrasonography can be helpful in defining either a mass lesion or laryngeal dysfunction, although laryngoscopy with histopathology is required for definitive diagnosis. Laryngeal paralysis is found much less commonly in cats than in dogs and can be congenital or acquired. Mildly affected animals can be managed with weight loss and avoidance of heat, humidity and over-exertion, however cats that display marked clinical signs require surgical treatment via unilateral arytenoid lateralisation.

Tracheal obstruction can be caused by neoplasia (lymphoma, adenocarcinoma, plasmacytoma) or by a granuloma (parasitic, fungal or foreign body). An intraluminal mass effect is generally evident on radiographs and a histological diagnosis can sometimes be obtained through tracheoscopy, although anaesthesia is challenging. Bronchoscopic debulking can provide some quality of life with a neoplastic obstruction, although resection and anastomosis is preferred for more definitive control.

\section{KEY LEARNING OBJECTIVES}

- Identify the site of obstruction based on clinical complaints and physical examination findings

- Generate a list of differential diagnoses for the cat with upper airway obstruction

- Prioritise diagnostic testing in the cat suspected of upper airway obstruction

\section{MULTIPLE CHOICE OUESTIONS}

1. Loud sounds heard without a stethoscope can be due to obstruction in any part of the respiratory tract except which of the following?
a. Nasal cavity
b. Nasopharynx
c. Larynx
d. Bronchi

2. Bilateral loss of nasal airflow suggests disease of which of the following?
a. Left or right nasal cavity
b. Nasopharynx
c. Larynx
d. Cervical trachea

3. Which fungal infection most commonly affects the nasal cavity of cats?
a. Coccidioidomycosis
b. Conidiobolus
c. Cryptococcus
d. Cryptosporidium

4. Which of the following disease processes is least likely to result in nasal obstruction?
a. Neoplasia
b. Fungal infection
c. Chronic rhinosinusitis
d. Nasopharyngeal stenosis

5. Which diagnostic test can be performed to distinguish laryngeal mass from laryngeal paralysis?
a. Radiography
b. Tidal breathing flow volume loop
c. Ultrasonography
d. C reactive protein

\section{Strategies for therapy of pneumonia}

\section{Lynelle Johnson}

Animals with bacterial pneumonia usually have a history of a productive cough, fever, tachypnoea and respiratory distress. However, some animals present with more vague signs of illness such as malaise, depression, anorexia and weight loss. Rapid or difficulty breathing can be noted by the owner. Thoracic auscultation is typically abnormal with loud or harsh lung sounds, and crackles can be variably detected. Fever may or may not be present. Mucopurulent nasal discharge can be observed when animals cough respiratory secretions into the nasopharynx or have coincident nasal infection.

A complete database will aid in establishing the severity of the pneumonia and identifying predisposing conditions. Pulse oximetry is a valuable tool for determining the severity of illness and also for following response to therapy. Alveolar infiltrates with air bronchograms are 